\title{
Exploring how gender figures the identity trajectories of two doctoral students in observational astrophysics
}

\author{
Allison J. Gonsalves \\ McGill University, Faculty of Education, 3700 rue McTavish, Montreal, Quebec H3Y 1A2, Canada
}

(Received 15 May 2017; published 15 June 2018)

\begin{abstract}
[This paper is part of the Focused Collection on Astronomy Education Research.] This paper presents the cases of two doctoral students in observational astrophysics whose circumstances and experiences led them on a career trajectory out of academic research. In this article, I employ a sociocultural lens that provides insight into the dynamics of students' experiences in astrophysics, which can in turn enrich our understandings of the lack of women in physics. I documented ethnographically two doctoral students' experiences in a physics department at a large research university in Canada. In turn, I employed an analytic framework of figured worlds, cultural models, and identity trajectories to understand the challenges these two doctoral students faced. I use data drawn from observational field notes, interviews, and participants' photo-narrative journals to explore the dominant cultural models of astrophysicist that were reproduced in their doctoral program. The analysis shows cultural models for recognizable astrophysicists in this doctoral program often did not fit neatly with these students' experiences, and at times interfered with their trajectories into astrophysics careers. Additionally, results indicate that a prevailing discourse of gender neutrality and the rejection of normative femininity in astrophysics both afforded and constrained participants' opportunities for recognition as insiders to astrophysics. However, the two participants repositioned themselves on alternative trajectories into physics teaching outside of academia, which had positive consequences for their astrophysicist identities. This study provides insights into the experiences of doctoral astrophysics that figure students' insider or outsider identities, and the role that gender plays in the shaping of those identity and career trajectories.
\end{abstract}

DOI: 10.1103/PhysRevPhysEducRes.14.010146

\section{INTRODUCTION}

The number of women participating in graduate-level physics in Canada is exceptionally low. Current statistical data puts the percentage of women in doctoral physics programs at around 19\% [1]. There are presently no recent national data in Canada that disaggregate physical science fields, but data from the U.S. indicate that women's enrollment in astronomy and astrophysics is slightly higher, at $35 \%$ for doctoral programs [2]. Despite astronomy being labeled as a gateway to physics, women do not seem to persist into astronomy or astrophysics faculty positions at the same rate as men, occupying about $19 \%$ of faculty positions in the U.S. [3]. A recent longitudinal study of astronomy graduate students (LSAGS) [4] identified attrition of doctoral women from astrophysics as a persistent problem and suggested that women differentially experience certain challenges in astrophysics. Importantly, in this

Published by the American Physical Society under the terms of the Creative Commons Attribution 4.0 International license. Further distribution of this work must maintain attribution to the author(s) and the published article's title, journal citation, and DOI. study sex did not operate as a factor directly affecting attrition. This indicates that women's reasons for leaving the field are complex, and that the pathways to leaving often involve multiple steps. Thus, we have much to learn from studying the experiences of doctoral students in the field-particularly those who leave. The LSAGS study showed that women differentially experience graduate supervision, and that women were more likely to report challenges with the "two-body problem" related to relationships and parenting in academia. Other recent research has also showed that women in graduate physics and astronomy programs were likely to experience sexism and microagressions, particularly in the form of gender role stereotypes, indicating that a culture of sexism still exists in physics and astronomy programs [5]. While these studies tell us much about the kinds of structural limitations that women encounter when they enter astrophysics, there is still much to learn about the experiences women have navigating the cultural elements of the field.

The two cases presented in this paper emerge from a larger study aimed at exploring the discourses about gender and competence that doctoral students constitute through their practice of physics [6]. Details of this study will be presented in the methodology section. Aspects of this study 
have been presented previously to explore the salience of masculinity as it emerges in doctoral physics culture [7], and to highlight the importance of recognition and the gendering of technical and analytical competence as critical components of "physicist identities" in doctoral work [8]. This paper complements previous work by providing a more in-depth examination of the two participants' different experiences within the contexts of their practices in one astrophysics research group. The stories presented here give us insights into the identity constructions of two women doctoral students in astrophysics through the useful concept of "identity trajectories" [9] to understand how their movement through a doctoral program also entails moments of greater and lesser identification with astrophysics. These stories thus highlight alternative trajectories through astrophysics that challenge the linear conception of the academic pipeline or academic ladder [10].

\section{A. Moving away from binary gender deficit models}

This study follows a recent call [11] for physics education research (PER) to move away from "binary deficit-based models" (p. 5) to understand women's experiences in physics, and instead to focus on the ways that gender may intersect with a variety of experiences that students have in the field. Danielsson [12] has argued that research on gender in physics has tended to focus on gender as a stable variable, a lens that tends to present gender as a woman problem, which can be treated with women-friendly solutions. Traxler and colleagues [11] have suggested that the prevalence of this approach has resulted in the implicit or explicit question "why can't women be more like men?" (p. 7). To move away from a research frame that seeks to change women so they can succeed in a culture where men are successful, it is necessary to take an approach to gender that does not rely on a binary understanding of what it means to be a man or a woman in a local culture. ${ }^{1}$

In this study, gender is understood as local and performative [13], and constructed through a "stylized repetition of acts" like behaviors, dress, speech, interests, and other enactments (p. 140). Thus, what is regarded as masculine or feminine or gender neutral, and how these are valued, is specific to the context of the local culture (e.g., a physics department). Importantly, Butler suggests the act of gender "that one performs is, in a sense, an act that's been going on before one arrived on the scene" [14], (p. 526). This signals that the conventions of gender exist and are rehearsed by actors. These conventions of gender are understood by the actors in the local context, and are

\footnotetext{
${ }^{1}$ To clarify terminology, in this paper I do not wish to conflate sex and gender. Sex refers to an individual's biological and physiological characteristics and is generally assigned (male, female, or intersex) at birth. Gender is an expression of identity that does not need to be connected to one's biological sex. Gender expressions exist on a continuum of masculinity and femininity, they are not fixed, and may change over time.
}

recognizable, in the forms of masculinity and femininity [15], as ideal typical forms. Paechter argues that masculinity and femininity are "actual ways that real people construct and understand themselves in terms of how they 'do' boy/man or girl/'woman'” (p. 262). I do not wish to posit that masculinities and femininities are signifiers of maleness or femaleness. These performances exist on a continuum and have various relationships to multiple forms of masculinities and femininities and can be taken up by individuals of any gender. An individual may perform a number or set of masculinities and femininities in various recognizable ways, in various local contexts. To illustrate, Traxler and colleagues [11] give several concrete examples of the local and contextual nature of gender performances. They discuss the social performance of wearing a dress as signaling femininity, which is usually associated with women. They suggest that in North American culture, "a man who wears pants and acts tough, competitive, and in a manner to be viewed as a leader (all stereotypically masculine traits) would most likely not come to his workplace in a dress." (p. 5). However, in a different cultural context, a similar man in Scotland (still wishing to perform stereotypical masculinity) might safely wear a kilt and still be recognized as "appropriately" performing gender in that context. Thinking of gender as performative, tentative, and contextual permits me to examine how doing astrophysics and doing gender are mutually constituted in a process of identification that involves convincingly performing an identity role that is recognizable in the local context of the physics department or astrophysics community at large. This perspective on gender reveals the complexities of gendered experiences in astrophysics environments, rather than framing women's experiences as unitary and predictable.

\section{THEORETICAL FRAMEWORK}

\section{A. Identity work}

In this paper, I understand learning as not only the acquisition of concepts, but also the construction of identities in the social contexts in which those concepts are acquired [16]. In a sociocultural approach to research, learning may be thought of as activity, and it is not possible to understand activity without understanding the context in which it occurs [17]. Similarly, these activities are shaped by discourses extant within in that context; in turn, discourses shape and are shaped by the identities of people engaging in activities [18]. From this perspective, learning to be an astrophysicist entails the concomitant construction of conceptual knowledge and of an identity as an insider to astrophysics [19]. Thus, rather than looking at differences between how men and women learn astrophysics, I am interested in how students learn to become astrophysicists, and the contexts in which learning to become happens. In this sense, I am examining doctoral students' identity work 
in astrophysics; that is, work which entails authoring oneself as a recognizable astrophysicist amid discourses that shape who one thinks they must be to engage in astrophysics $[20,21]$ or to be identified as an "astrophysicist." A focus on identity work has the promising feature of moving away from restrictive binary conceptions of gender that are often grounded in deficit perspectives that frame female students as lacking some component necessary to persist in physics (i.e., self-confidence or spatial-reasoning skills) [11]. This perspective permits the examination of a more fluid range of gender identities, and how these intersect with a range of astrophysicist identities. The analysis is also shifted from identifying structural factors (e.g., mentoring [5] or departmental support [22]) related to women's persistence in the field, to the subtle ways that gender can influence students' beliefs about who they must be to engage in astrophysics culture.

Carlone and Johnson present a useful model for understanding the identity work that doctoral astrophysics students engage in Ref. [23]. According to them, science identities may be formed when one decides to be a "certain kind of person" [24] and then carries out activities that may gain recognition to that effect. This model is particularly useful for this study, as it was developed out of an examination of the narratives of women of color as they learned how to become scientists across different domains of science in post-secondary institutions. Carlone and Johnson argue that beneficial forms of recognition often come from meaningful others and can shape how a person perceives themselves in relation to the field. This perspective is particularly resonant now, as it reflects recent trends in PER that highlight the salience of recognition and identification to persistence in the field [23,25,6-9]. Like this study, Carlone and Johnson used interviews as the primary data source, thus relying on participants' stories of positive or negative recognition to understand how they author themselves in a particular context [23]. These moments of recognition, as narrated by participants, are salient to their identity work at the time. Carlone and Johnson argue that these identity constructions, which occur at a moment in time, may change or be interpreted differently at later moments and in different contexts. Danielsson $[12,26]$ has also applied this conception of identity to interview data to demonstrate how undergraduate physics students construct identities as they simultaneously "do physics" and "do gender." Similarly, Johnson and colleagues [27] have foregrounded identity work to study "how individuals negotiate settings and how they are understood and responded to by more established members of settings" (p. 344), and have privileged women's narrative accounts (through interviews and emails) to do so.

\section{B. Identity trajectories}

In this paper, I conceptualize identity work over time as identity trajectories [17,23] to explore the shifts and transformations that may occur over time as participants move through their degree programs. I draw from Jackson and Seiler [17] who, using primarily narrative data from interviews and reflective journals, have studied the identity trajectories of latecomers to science and have explored how trajectories are formed as stronger or weaker identification to science fluctuates over time. They argue that trajectories do not imply linearity, but that there is a momentum in relation to science that occurs as a "buildup of resources that creates a patterning or thickening of identity" (p. 831). Taking up this dynamic conceptualization of identity, I explore the trajectories of two women astrophysicists as they navigate stories of what it takes to be recognized as a competent member of the astrophysics community. Jackson and Seiler's use of the concept of identity trajectories is particularly appropriate for this study, as their reliance on participants' self-authoring through narratives of identity rather than identity in action generated "insights into another aspect of identity work that continues after an experience takes place, but still represents students' attempts to author themselves in relation to science" (p. 850). This perspective helps us to see identity work as it happens over time and gives us some insight into how participants interpret events and make choices in response to them.

\section{Figured worlds}

The figured worlds [28] of astrophysics are repeatedly constructed and reconstructed by the prevailing storylines and images of how to appropriately perform and be recognized as an acceptable or "good" astrophysicist. Figured worlds are the

socially and cultural constructed realm[s] of interpretation in which particular characters and actors are recognized, significance is assigned to certain acts, and particular outcomes are valued over others [28], (p. 52).

Gee [29] argues that figured worlds can be thought of as individuals' taken-for-granted theories about the way things are. They are the stereotypical first impressions and the simplified or unconscious stories about the way the world works. Figured worlds may be thought of as the storylines about being a recognizable astrophysicist that provide the backdrop against which students learn to become astrophysicists-since being an astrophysicist in a way that is culturally recognized involves more than simply doing experiments or solving theoretical problems. In doing so, the figured worlds of astrophysics can construct identity positions by defining the appropriate content and practices for astrophysicists. As such, they give a sense of what it is possible, right, and acceptable for individuals to do and be recognized as astrophysicists [28]. 
The concept of figured worlds is employed as a useful heuristic to construct a local and contextual understanding of what doing astrophysics entails, while positioning this local understanding in the larger context of physics, gender, and academia. Conceptualizing astrophysics cultures as figured worlds permits an exploration of actors' participation in identity work in the context of available resources and cultural models in astrophysics. Identity work in this case entails the work that actors do to gain recognition as competent astrophysicists. Individuals make bids to be recognized as a certain kind of person, thus taking up a storyline of astrophysicist in an appropriate and acceptable way [29]. Successful bids may be how students begin to think of themselves as astrophysicists and they may consequently make similar bids to pursue that kind of subject position in the future.

Recognition often depends on successfully mobilizing available resources and cultural models in the figured world. Cultural models "consist of schemas (mental or emotional knowledge structures) that guide attention to, draw inferences about, and evaluate experience" [28] (p. 297). They may also include or draw on available resources in the forms of material objects, text, language, behaviors, and styles of dress [28]. In the figured worlds of astrophysics, some of the recognizable cultural models have been constructed in broader cultural contexts (e.g., positions related to race, gender, and class). Others might be more local cultural models with meanings specific to the astrophysics community, for example, images of geeky physicists [8,30], how to be an academic, or even acceptable cultural models of academic participation in a particular university department. Although cultural models may be constraining to actors, they may also offer opportunities for actors to mobilize resources in new and improvisational ways to carve out opportunities for recognition in the figured worlds of astrophysics [28]. Following Jackson and Seiler, it is understood that actors can narrate or author who they are in relation to the cultural models of the figured world in ways that may be regarded as "retelling and reinterpretations of events and identity formations" [9] (p. 829). In listening to and retelling these accounts of identification, it is understood that these are stories about how participants figure themselves in relation to the figured worlds of astrophysics. The terms figuring and authoring are used analytically to describe how actors' identities shift (or are refigured) in relation to the figured world, and how the actor interprets and represents these events $[28,9]$. These figurations over time may constitute trajectories of identification [9,21], where participants may form stronger or weaker identification with astrophysics at various times and various places. Thus, the salient analytical categories for this article are the stories of identity work that participants use to figure themselves as recognizable astrophysicists.

The research question used to guide this study was: How are identity trajectories of two women doctoral students afforded or constrained by the resources and cultural models available in the figured world of astrophysics? This question considers that identity trajectories depend on mobilizing the resources associated with cultural models to position oneself as a recognizable person in the figured world of astrophysics.

\section{METHODOLOGY}

\section{A. Case studies}

This article presents two case studies of doctoral astrophysics students that emerged from a larger ethnographic study conducted in a physics department at a researchintensive university in Eastern Canada, referred to here as Eastern University. ${ }^{2}$ Over the course of one year, field observations and interviews were conducted with 11 men and women across a variety of disciplinary subfields in the department. The men and women were all doctoral students at various stages in their degree process. As the principle investigator, I spent significant time with all the participants in their offices and labs, at seminars and meetings, and at social events. My interest in the various facets of graduate life that impacted participants' identity trajectories emerged from my own experiences as a graduate student in the natural sciences. As a former science graduate student, but coming from a different discipline, I could act as both an insider (knowing the many conventions and expectations of graduate science studies) and an outsider, observing participants in a discipline that I was less familiar with. As such, this research emerges from questions I deemed were absent in the field of astrophysics education research, and from my own experiences in graduate studies. I understand my subjectivity in this research to contribute to the data collection and analysis, as it shaped the questions I asked and the care and responsibility I assumed for the responses. To make claims about their life experiences, I took care to dialogue with participants about the way I told their stories, and I offered multiple opportunities to feedback into the writing process to help me faithfully represent their stories.

The two case studies offered here center on the experiences of Ruby and Molly, ${ }^{3}$ who were midway through their doctoral program at the time of this study. These two cases were chosen for case analysis as they represented contrasting experiences on trajectories to becoming astrophysicists. Ruby and Molly were both recruited to the study using purposeful sampling [31], and both agreed to participate in all forms of data collection. Molly was a white, Canadian born woman in her mid-20s who was pregnant with her first child at the time of the study. Ruby was of Middle Eastern origin and had moved to Canada by the second year of secondary school. She was also in her mid-20s. Both participants worked in the same research

\footnotetext{
${ }^{2}$ Eastern University is a pseudonym.

${ }^{3}$ Ruby and Molly are both pseudonyms.
} 
group, the StarLab, ${ }^{4}$ which was led by a prominent woman astrophysicist.

\section{B. Data collection}

Multiple data sources were collected over the course of one year. These include field observations, ethnographic field notes, journaling, photo-elicitation interviews, and semistructured interviews. This study uses a novel interviewing methodology known as photoreflexivity [32-34] to elicit stories of identification-stories where participants identified themselves as engaging in astrophysics learning in such a way that rendered them recognizable as astrophysicists - both to themselves and to meaningful others in the field [23]. Participants took photos of aspects of physics culture that was important to them and kept them annotated in a journal. The photos were used as jumping-off points in interviews in the process of photo-elicitation [35,36]. Two interviews were conducted with each of the participants, the first involved photo-elicitation, and the second a semistructured interview elaborating on points raised in analysis of the first interview [37,38]. The case studies presented here report primarily on evidence collected from photoelicitation and follow up interview data.

\section{Data analysis}

Data analysis proceeded in a three-part format to first fashion narratives of Molly and Ruby's histories with astrophysics, to characterize their identity work in astrophysics, and to identify the cultural models for astrophysicists prevalent in this local context.

I analyzed interview transcripts to construct personal histories that were fashioned into storylines using Mishler's [39] life history narratives approach. To do this, I coded narrative sections (important stories) to create identity trajectories highlighting specific markers that impacted the participants' movement through doctoral astrophysics and beyond (for example, showing an early interest in physics, having an influential teacher, experiences in undergraduate physics, reasons for selecting graduate studies in astrophysics, encouraging or discouraging experiences as a doctoral student). These data points were compiled into life history narratives that discussed further in the case studies presented in the following sections.

To characterize Molly and Ruby's identity trajectories, I approached each narrative section of data using Gee's [29] "identities building tool" and asked of the data "What socially recognizable identity or identities [is] the speaker trying to enact or to get others to recognize?... What sorts of identities [does] the speaker recognize for others in relationship to her own?" (p. 110). Data were pieced into episodes where participants authored themselves as astrophysicists through stories of engagement in science as a child, as an undergraduate, and then as a doctoral student

\footnotetext{
${ }^{4}$ StarLab is a pseudonym for the research group that both Molly and Ruby were members of at Eastern University.
}

and beyond. These stories permitted the construction of identity trajectories that moved through various stages of becoming astrophysicists: (i) entry into astrophysics; (ii) stories of recognition (either when a participant recognized herself as being a competent astrophysicist or when she was recognized by others in both positive and negative ways); and (iii) moving forward.

I then conducted a third level of analysis to characterize the figured world of astrophysics. To identify the cultural models for astrophysics that are constructed in the field, I used Gee's [29] "figured worlds tool" to examine narrative sections for characterizations of appropriate ways of doing astrophysics and being astrophysicists. This entailed looking for episodes in the data where participants discussed appropriate ways of acting, interacting, talking, writing and doing research, communicating and dressing, as well as appropriate beliefs and values $[29,40]$. I did so by approaching each narrative section of data with the question: "What typical stories [are] the words and phrases of the communication assuming and inviting listeners to assume? What participants, activities, ways of interacting, forms of language [etc. are in these figured worlds?" [29] (p. 171). I coded instances where cultural models of astrophysics intersected with the participants' authoring of herself as a recognizable astrophysicist, and then I asked the following questions of the data: how is identity work enabled or constrained by the cultural models of astrophysics, and what available resources does the participant mobilize to position themselves as recognizable in the figured world of astrophysics? [9]

This process yielded two predominant cultural models for astrophysicists: competence and the stereotypical physicist. The third stage of the analysis also permitted an examination of the kinds of resources available in the figured world of astrophysics, which participants could mobilize to author insider identities to astrophysics. I describe cultural models and resources in Sec. IV. A. I then went back to the stories of identification to examine how these cultural models and associated resources may have impacted the participants' identity trajectories, to create the case studies presented in Sec. IV. B.

\section{FINDINGS}

The findings from this study provide examples of the very complex identity work that women do to gain recognition as competent participants in this local figured world of observational astrophysics. Evidence ${ }^{5}$ provided

\footnotetext{
${ }^{5}$ As there are several sources for data in each case study (interviews, photo-journals, field notes), I have indicated the source code for each piece of data (INT = interview, PJ = photo-journal, $\mathrm{FN}=$ fieldnotes), along with cataloguing codes. Transcriptions are presented verbatim with syntax errors indicated as [sic], and pauses in the transcript as ellipses [...]. In instances where the author has taken the liberty to substitute words or phrases to fit the transcript into the surrounding text, the edit is marked with a square bracket [].
} 
here emerges primarily from photo-elicitation and semistructured interviews. This section is structured to first present an overview of the figured world of astrophysics and the dominant cultural models and resources that emerged from the analysis. Then case studies of the participants, Ruby and Molly, are presented to illustrate how the cultural models and available resources in the figured world of astrophysics shape their identity trajectories.

\section{A. Cultural models in the figured world of astrophysics}

Emerging from this analysis are two dominant cultural models for astrophysicists around which doctoral students authored themselves. At times these cultural models enabled participants to author themselves as recognizable astrophysicists, and at times these cultural models constrained their authoring. These cultural models-the cultural model of competence, and the cultural model of the stereotypical astrophysicist-limit the possibilities for students to do identity work and to position themselves as recognizable astrophysicists. I describe these cultural models below, and then mobilize them to frame students' stories of experience in the case studies presented afterwards.

\section{Competence}

When participants discussed doing astrophysics or ways of recognizing astrophysicists, it was usually in relation to a form of competence. Carlone and Johnson have previously described competence as a feature of science identity that can be identified and measured by grades [23]. However, in doctoral studies, competence is also likely to be assessed by productivity (conference presentations, publications, collaboration). I refer to this as academic competence, ${ }^{6}$ which participants constructed as "knowing" the field, being able to construct careful arguments or to deconstruct others' arguments, productivity, and good communication skills. Competence provides a framework for recognizing "good astrophysicists," around which students may be positioned in various ways. In the figured world of astrophysics, collaboration becomes a very important resource to developing academic competence. This is reified through resources like the Star Tea and the Cosmic Coffee events, ${ }^{7}$ and in artifacts like multiple author publications. Communication and argumentation skills are developed in these collaborative meetings, as well as through research group meetings

\footnotetext{
${ }^{6}$ Detailed accounts of the gendering of technical and analytical competence emerging from this study have been reported elsewhere $[6,8]$.

${ }^{7}$ The Star Tea and Cosmic Coffee are discussion-based meetings where graduate students, post-docs, research associates, research assistants, work-study students and professors meet to discuss recent publications in their research fields. These are pseudonyms for the regular meetings, which are ongoing at Eastern University.
}

and presentations at conferences. These events therefore serve as resources to support identity trajectories into academic careers. Becoming skilled at argumentation and communication is critical to the figuring of a recognizable competent astrophysicist identity.

\section{Stereotypical astrophysicist}

In this study, the stereotypical astrophysicist emerges as a complex construct that includes various seemingly contradictory elements. The cultural model for the stereotypical astrophysicist was described primarily in terms of appearance and behavior: always male, somewhat disheveled, and often wearing a physicist uniform which was dependent on disciplinary context. In this case, participants constructed astrophysicists as "always wearing sandals with socks and usually a t-shirt from the telescope they are working at" (INT.01A). However, despite descriptions of astrophysicists as primarily male, a central feature of the cultural model of the stereotypical astrophysicist are the notions of gender neutrality [41]—as one participant described "we're all unisex anyway" (INT.01C), and gender normativity, which positions stereotypical femininity [42,15] (described by Ruby as 'girly girls') outside of physics [8].

\section{Resources present in the figured world of astrophysics}

In the figured world of doctoral education, students are often expected to participate in activities that will increase their likelihood of gaining recognition in the field (such as publishing, attending conferences, summer schools, lab or research group meetings, departmental gatherings and seminars), which may lead to postdoctoral study and ultimately researcher or faculty positions. These activities act as resources for identification, and doctoral students have variable access to these resources. Examples of the resources available in the figured world of astrophysics at Eastern University are the weekly research team meetings (led by the PI, who supervised 11 graduate students at the time); the biweekly Star Tea and Cosmic Coffee meetings. It is expected that students aspiring to astrophysics doctoral degrees will avail themselves of these resources early on. Often, getting into a doctoral astrophysics program and being selected to be a part of a prestigious research group like the one Ruby and Molly belong to entails work study in a research group as an undergraduate. It is common in this research group for students to become engaged in research projects as undergraduate students and persist through to the doctorate, at which point they are generally seen as more experienced members of the community and may even take lead author on research reports that are published in prestigious research journals like Nature. This kind of positioning for a doctoral student entails making use of several different kinds of resources: collaboration with peers in the research group, attending research meetings, developing writing and research skills, staying on top of 
current research by reading all the most recent publications, and attending seminars and reading groups.

\section{B. Case studies}

This section presents summaries of the identity trajectories of two astrophysics doctoral students, Ruby and Molly. These two participants were selected as they provide alternate stories to the usual identity trajectories expected for doctoral students in their field. These cases are not illustrative of attrition from the field. Both Ruby and Molly completed their doctorates and worked for a time as researchers with their research groups. However, both elected to pursue college teaching instead of academic research. These identity trajectories aim to make sense of how engagement with the cultural models of astrophysics impacted their ability or desire to author identities as astrophysicists.

\section{Ruby}

Ruby's interview data present a rich and complex identity trajectory that begins with an early fascination with astronomy, the authoring of herself as a competent physics student, and ultimately a disillusion with the dayto-day practices of astrophysics. These movements can be mapped on to Ruby's disengagement with the cultural model for competence and improvisation with the cultural model for stereotypical physicists.

(a) Entry into astrophysics. Ruby's trajectory through the figured world of astrophysics entails numerous identity refigurings. Her life history interviews demonstrate a move from finding congruence with the cultural model of competence, while later coming into conflict with it. Ruby's early interest in astrophysics developed through a fascination with the night sky. She describes how she came to love astronomy through her exposure to French comics like TinTin:

"[O]ne day [my father] just sat next to me and opened TinTin l'étoile mystérieuse and he read it to me and I was looking at all the pictures, you know, and felt awe. I sort of got interested in things in the sky I guess. So, it wasn't really physics, it was really things in the sky." (INT.01)

Ruby then described how she learned in high school that she would need to take physics to study the stars and "around the time I think I was taking physical sciences or something in high school and I thought, oh that fits perfectly, physical science is my favorite." (INT.01). For Ruby, at this point, the figured world of astrophysics was constructed by romantic ideas of studying stars and looking at the night sky. These expectations, along with high school physics teachers who were enthusiastic, recognized her ability, and encouraged her to study physics, acted as resources to position her on the trajectory into studying physics at Eastern University, where she excelled throughout her undergraduate degree. By all accounts, Ruby entered the figured world of astrophysics with a significant amount of science capital ${ }^{8}$ [43] and a recognizable insider identity. Ruby narrated her early astrophysics identity and positions herself as having natural ability. Natural ability is a part of the larger cultural model of competence, where as an undergraduate she was recognized as the kind of person who would be repeatedly stopped by other students to ask for her notes or to ask her questions. She suggested that she graduated "at the top of [her] class" (INT.01), which she credited to a natural ability for problem solving and the fact that in physics "every problem has an answer" (INT.01).

(b) Stories of recognition. By the midpoint of her doctoral program, however, Ruby's orientation to the figured world of astrophysics has shifted considerably. She still authors herself in relation to natural ability, but now positions herself oppositionally. Ruby described her struggles to recall basic concepts throughout the course of her doctoral study, saying "I forget everything, I swear" (R.INT.01). She also described being disillusioned by the reality and day-to-day mundanity of doctoral astrophysics:

[I]t is not what Tintin and his friends were doing. It is, now I don't even do optical astronomy, so the rays, the rays that the stars emit that I analyze, you can't see them with a naked eye, you need an $x$-ray detector [...] I think I had this romantic ideal, 'oh I will sit in front of a telescope and look at stars and we can see the satellites and Jupiter'. Nobody looks at satellites and Jupiter any more. (INT.01)

This disillusion led Ruby to stop participating in the collaborative events that most doctoral students used as resources for sharing knowledge about recent research, and for positioning themselves as insiders to the figured world of astrophysics. In addition, Ruby talked about the emphasis placed on productivity, which is often enabled by the resources provided through collaborative opportunities like research group meetings, the Star Tea or the Cosmic Coffee. She described the life of doctoral students in the StarLab as highly productive: "In the StarLab, they are all like really motivated, and they read lots of papers, and they certainly know a lot more physics about the stars that we are looking at than me" (INT.01). In the photo-elicitation interview, Ruby showed a photo of the seminar room,

\footnotetext{
${ }^{8}$ Science capital is a sociological concept proposed by Archer and colleagues in 2015 to extend the Bourdieusian concepts of social and cultural capital, where social capital refers to social networks and relations, and cultural capital refers to qualifications, dispositions, and cultural goods. Science capital refers specifically to science related forms of social capital (e.g., parental scientific knowledge, talking to others about science), and science related forms of cultural capital (scientific literacy, science dispositions, symbolic forms of knowledge about the transferability of science qualifications).
} 
where collaborative meetings and reading groups take place (PJ.04). ${ }^{9}$ Looking at it, Ruby said:

The one I don't go into yeah (laughter) well yeah 'cause it makes me feel incredibly guilty, that is why it gives me a bad feeling [...] Yeah, like I feel so out of place when I sit in a place with you know, the other people from the group. They all know so much more than me. (INT.01)

Here, the emphasis on collaboration, argumentation, and productivity that emerges in the cultural model of competence positions Ruby as an outsider. By these measures of participation, Ruby does not author herself as an insider to astrophysics. However, despite her self-positioning in opposition to the conventional cultural model for academic competence in the field, Ruby still maintains identification as an astrophysicist.

(c) Mobilizing resources to author an insider identity. Of note in Ruby's story is the very strong stance that she took on the gendering of astrophysics or physics in general. When I first invited her to participate in the study, Ruby claimed that she might not have much to "contribute" to the study, because she did not think that gender was a problem in studying physics (FN.07.15.11). She asserted, "I guess I just don't think of it as that big of an issue, you know like, the women in physics thing, people make it a very big issue as well and I just don't think it is a very big issue at all" (INT.02B). In fact, Ruby said she "liked it" that there were very few women in physics when she was an undergraduate. Ruby's identity trajectory through astrophysics was often characterized by a distancing from stereotypical femininity [15], where she enjoyed being one of the "few girls" (INT.02A) in physics courses. In this way, Ruby authors herself as a stereotypical physicist, by asserting her difference to other women.

Interviews and discussions in the field with Ruby often turned to descriptions of acceptable gender performances within the context of the community of astrophysics. During our interviews, Ruby positioned herself as a recognizable astrophysicist by suggesting that she looked like a stereotypical one:

I don't know why that is but I have a feeling that physicists, for some reason that I don't understand, they allow, they don't mind when their personality quirks show. If you look at the physics building almost every astro person is wearing sandals. You even hear people say it, like um, there is an astro prof at the University on the Mountain ${ }^{10}$ who um, we were on our way to [Astronomical Society Conference] last year, he said, 'they will recognize us by our sandals' [as] the group of

\footnotetext{
${ }^{9}$ Ruby did not grant permission for her photos to be available for publication.

${ }^{10}$ University on the Mountain is a pseudonym for another Eastern Canadian University.
}

physicists arriving, or astronomers or whatever. Sometimes, I speak of them as interchangeable. So, um, so yes, sometimes wearing sandals makes me think, feel like a physicist. (INT.02A)

By authoring herself in relation to this cultural model, Ruby not only positioned herself as a recognizable astrophysicist, but also positioned herself against the so-called girly girls who "wear high heels and speak with the word 'like' all the time and giggle at nothing and [...] spend an hour every single day on their appearance." (INT.02A) When probed about whether girly girls might be successful in astrophysics, Ruby concedes,

Yeah, a few that I run into in the corridor. I don't understand how you could combine being a physicist and a girly girl, but they kind of combine it [...] I am not saying they are doing something wrong. (INT.02A)

This discursive incompatibility between the girly girl and the physicist has been identified elsewhere [44], and by positioning herself in opposition to the girly girl, Ruby aligned herself with the cultural model for stereotypical physicist. Ruby also suggested that there is a tension between women who prioritize taking care of their appearance and doing physics. In doing so, Ruby rejects stereotypical femininity [16] aligns herself with a masculinized cultural model [45]. Paechter [16] has suggested that a distancing from stereotypical femininity is a claiming of power, or a rejection of the disempowerment that comes with this normalized form of femininity. Ong [46] reported that women learning physics often take up masculinized traits as strategies for "gendered passing." Others have studied how students often author themselves as "one of the boys" as a strategy for identification in physics and engineering $[12,47]$. By adopting these strategies, Ruby improvises around the cultural model for stereotypical astrophysicist to author herself a recognizable identity in the figured world of astrophysics. She does this despite her relative isolation and failure to gain recognition in relation to the cultural model for competence.

(d) Moving forward. As she nears the end of her doctorate, Ruby has authored for herself a new identity in relation to the figured world of astrophysics: a teacher. Ruby took on a job teaching college-level physics while she still engaged in astrophysics research as an associate researcher. The activity of teaching physics helped Ruby to position herself as a recognizable astrophysicist:

When I can explain what is around me I sort of feel like a physicist. When I, when I teach I feel like a physicist because I know more physics than the students I teach... Yes, when I can explain what is going on around me, yes, when I am teaching physics [I feel like a physicist]. (INT.02A) 
The expected model for participation in astrophysics often ends at the level of the researcher or academic scientist. However, Ruby's story demonstrates that it is possible to author an identity as an astrophysicist outside of the usual means of recognition. Ruby's narrative details a trajectory that would normally be peripheral or outbound to a typical doctoral astrophysics route; despite this, Ruby finds ways to author herself as an insider to astrophysics. This contrasts with Molly's trajectory, which might be characterized as a more stable inbound route.

\section{Molly}

Molly narrated her trajectory as a consistently increasing identification with the figured world of astrophysics. Her case is one of a doctoral student who makes use of all the resources available through the cultural model of competence, but even so, she described complex identity refigurings in relation to gender and the cultural model of stereotypical astrophysicists.

(a) Early entry into astrophysics. Molly's life history narrative does not begin with the same kind of early fascination with astrophysics that Ruby details. Molly grew up in a household that emphasized art. Her mother was a ballet teacher, and Molly talked about being brought up in the ballet school. Ballet took precedence over other studies during her youth, but despite this, Molly still arrived at physics with a great deal of science capital [43]. Her father had been a research scientist and encouraged her to develop an interest in science. While in high school, a physics teacher recognized her aptitude for math and science and encouraged Molly to pursue an engineering degree. Molly enrolled in an undergraduate engineering program but was quickly discouraged after a work-study placement in the summer following her first year. Citing engineering's emphasis on business rather than science, Molly switched programs to physics. In the third year of her undergraduate program, Molly transferred to Eastern University. During the following summer, she took a position as a summer researcher in the StarLab. She went on to do her Master's degree and Ph.D. with the same supervisor. She described the experience of working in this research group as very supportive, and early experiences gaining recognition at conferences as significant to her identity work in astrophysics: "People were interested in my work and I got compliments on it. Definitely I felt like a part of the community" (INT.02). Like Ruby, Molly does not explicitly regard gender as an important issue emerging in her experience in doctoral astrophysics. Although she told stories where gender and gender issues figure prominently, being a woman and being an astrophysicist was never problematic for Molly: "There has been nothing that I've encountered that has been discouraging to me as a woman. [...] I've had nothing but encouragement." (INT.02) (b) Stories of recognition. Interviews with Molly revealed that when she initially arrived at Eastern University, she stood out from the rest of the physicists in her department.

Um, at the beginning I feel like it was harder for me to make friends when I was a little punk rock girl. I definitely felt like everyone was looking at me like: 'who are you and why are you here?'[...] I was dying my hair weird colors which is extremely [unusual], and dressing really weird [...] Just dressing sort of outside the norm especially for physics, having bright orange hair, or blue hair. You're very visible, everybody knows who you are. (INT.01)

Additionally, Molly described only peripherally participating in departmental activities, as she focused on a social life outside of the department. In interviews, Molly thus authored herself in opposition to the cultural model of the stereotypical astrophysicist. At times, however, she was also positioned by others in opposition to this cultural model. She described being the only woman in one of her classes:

There was one class I took where I was the only woman. That was weird. I definitely had some weird experiences where I was the only woman and I felt like I was the only woman and everybody else noticed. There were comments that, just on fact that you're the only girl here. (INT.01)

Recent research on the experiences of women in physics departments points to the microaggressions that women often encounter when they are in the minority in a physics culture [5]. Some of these microaggressions materialize as assumptions of inferiority, which Molly experienced on more than one occasion:

I was working on this assignment with this guy and this guy said, I can't remember exactly what he said because it's a lot of years ago, but he basically said something like, 'Oh, do you find this class hard?' And I was like, 'yes!' because it's a very difficult class. 'Yes, it's hard.' He was like, 'you must find it particularly difficult because women can't visualize in three dimensions.' I can't? I didn't know that about myself! I was so flabbergasted by -he wasn't-he didn't realize that it was offensive. (INT.01)

Molly described this episode as "the first overt thing that anyone has ever said to me. I had never encountered anything about the fact that I was a woman. I would interpret people being weird because I looked different, or whatever" (INT.01). However, later she recounted a professor's reaction to her presence in his class, which was specifically for honors students in physics. "When I first 
arrived he asked me, 'oh, you're new here?' 'cause it was a third year class. He was like, 'you're in the honors program?' sort of like that. I was like, 'yeah.' He clearly was a little surprised" (INT.02). At times, Molly attributed people's reactions to her presence in the department to sexist microaggressions, but at other times, she suggested that she likely stood out because she did not fit in with the normative cultural model for the stereotypical astrophysicist.

At the time of this study, Molly was pregnant with her first child. She was the first graduate student in the physics department to be pregnant, and she described the reaction of one of her colleagues:

There were a bunch of profs that I know well enough to say hi to and chat with, one in particular that I had spoken with many times both about research stuff and more socially. He stopped saying hi to me completely. He still hasn't said hello to me since I've been pregnant. He doesn't make eye contact with me anymore. (INT.02)

In relation to the cultural model for the stereotypical physicist, Molly's gender performances positioned her as (in)visible. Faulkner [48] discusses the "(in)visibility paradox" (p. 181) wherein authoring oneself in opposition to normative identity positions in a field can make an actor both visible and invisible. In relation to the cultural model, therefore, Molly's pregnancy rendered her hypervisible, but unrecognizable as a stereotypical astrophysicist. Where Molly first subverted the cultural model for the stereotypical physicist by dyeing her hair different colors, she later stood out for being pregnant:

You really can't ignore how much of a woman I am now that I'm about to give birth, really obviously. Suddenly they really see me as a woman whereas before I was just another grad student. (INT.02)

Kvande [49] has argued that by being pregnant in the workplace in science-related fields, women erase the idea of gender neutrality and become more visible in very particular and gendered ways. Cisgendered ${ }^{11}$ motherhood puts an end to images of women in physics as one of the boys [12] or as part of a gender-neutral [8] community. For Molly, this meant the need to author a recognizable identity as an astrophysicist through other means. Indeed, this episode illustrates Faulkner's discussion of how fitting into a masculine culture like physics as an extra layer of work that women must do to be seen to belong to the community.

\footnotetext{
${ }^{11}$ Cisgender is a term for people whose gender identity matches the sex they were assigned at birth. 'Mother' is often assumed to be an identity exclusive to cisgendered women-prefacing 'motherhood' with the term cisgendered signifies an understanding that pregnant people may also have trans- or non-binary gender identities.
}

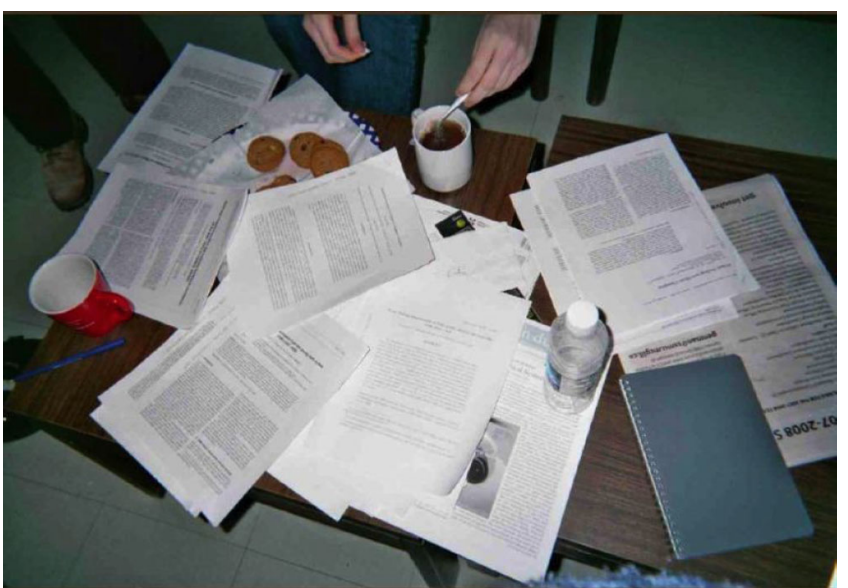

FIG. 1. Molly's photograph of the seminar room during a Cosmic Coffee meeting.

(c) Mobilizing resources to author an insider identity: Molly authored herself as a recognizable astrophysics doctoral student by leveraging the resources that were made available through the cultural model for academic competence. Molly described a strong onboarding process when she joined the StarLab:

I was extremely lucky. I had this office with a post-doc and it was really great for my life in general, being in the physics department. I had access to computers where I could do all my research and type everything up, do all that. The post-doc was really helpful in terms of just chatting about general [star ${ }^{12}$ ] stuff. When you come in, it takes a while to really understand all that's going on. He was super helpful. The group is very friendly so you get to know people better. I guess I started off sort of shy in that situation. Everyone knows more than you, everyone's a grad student or a post-doc. You're pretty quiet at the beginning. Once you sort of realize that I'm not, I'm a bit further behind everybody else, but there's no fundamental difference between us, I started getting more confident and able to ask questions. (INT.01)

The resources that Molly detailed here are critical to demonstrating competence through productivity and collaboration. Like Ruby, Molly also took a photograph of the seminar room (Fig. 1), but unlike Ruby's, rather than an empty room, Molly's photograph was of a table covered in journal articles. Molly explained that this environment was where doctoral students learned how to communicate about physics:

It's definitely where you learn how to make arguments about stuff. I don't know if it's where you learn what's

\footnotetext{
${ }^{12}$ To ensure participant confidentiality, the topic of study in the "StarLab" has been given a pseudonym.
} 
good science and what's bad science, maybe a bit, partly. I think you get a little more of that just from reading a ton of papers and getting a sense for it. Definitely learning how to communicate about physics, which is super, super important and not something that's a part of the undergrad at all. [...] A lot of people in this department are super, super shy and don't hang out with a lot of people, so they don't even talk to a lot of friends in big groups. (INT.01)

Field note data indicate that Molly was often present at these meetings and a frequent contributor to the discussion (FN 07.04.18). By leveraging the resources made available through the cultural model of competence, like these frequently held collaborative meetings, Molly authored herself as a recognizable member of the figured world of astrophysics.

(d) Moving forward: Molly went on to do a post-doc in the same research group, and authored numerous publications. However, despite her ambition and considerable success, Molly's identity trajectory into astrophysics shifted as a result of her pregnancy:

I am less interested in what I'm doing at work right now because there's suddenly something else in my life that requires you to be passionate but also it's very easy to be excited and very passionate about becoming a mother. [...] Definitely for a few months I'm going to be chilling out but after that, I don't know. Again with the leaky pipeline thing, it seems that from what I've heard, that's when a lot of women back off. It also seems to have something to do with the fact that women in science tend to be married to men in science whereas men in science tend to be married to whoever. Typically, if there has to be someone who backs off, it tends to be the woman. (INT.02)

At the time of these interviews, Molly described herself as being "definitely the one that is the more ambitious" partner in her relationship and "the one who has written more papers and gone to more conferences." "I'm more involved in my physics community than he is in his," she said. "I'm much more likely to really push for a Harvard post-doc than him." (INT.02) However, after having a second child and completing a post-doc, Molly's career ambitions changed. Molly has since moved away from academic research, but still engages in physics as a college-level physics teacher.

\section{DISCUSSION}

These two case studies provide a new perspective on how identity trajectories are constituted in astrophysics as students move through doctoral degree programs. Importantly, these cases demonstrate two examples of the heterogeneity of experiences that women may have as they engage in the figured world of astrophysics. Using a theoretical framework that explores how students navigate the cultural models that shape the figured world of astrophysics, these stories demonstrate how gender can figure into students' experiences in very different ways. The data presented here give a more nuanced portrait of disciplinary and culturally specific constructions of gender and identity in relation to astrophysics education and practice. This helps to provide some much-needed perspective on the experiences women have as they navigate the astrophysics pipeline, and the strategies they may take to be recognized as insiders to the field.

This discussion section will provide some perspective on the identity trajectories mapped out in Ruby's and Molly's stories. First, I will consider the impact of the gendering of cultural models in the figured world of astrophysics. I will then address the issue of the astrophysics pipeline and discuss the need to reorient our thinking about what counts as persistence in astrophysics. Finally, I will look at how the concept of identity trajectories can provide perspectives on students' experiences in astrophysics that a pipeline orientation cannot, and I will consider future directions for research in this field.

\section{A. The gendering of cultural models}

While gender is not an explicit part of the narrative across the entirety of these participants' experiences, it is nonetheless possible to see how gender influences the ways that students author themselves or are positioned as they make bids for recognition in the figured world of astrophysics. Both women in the case studies presented here argued that their experiences were not shaped by gender, and that gender was "not a problem" for them in their own personal experiences in astrophysics. This ambivalence to gender in the framing of their experiences has been reported elsewhere [12,50]. For example, Walker [47] suggests that the overwhelming rejection of gender as an explanatory framework for women's experiences points to processes of normalization, rather than evidence of gender equity at work.

The gendering of the cultural model for the stereotypical physicist bears notable impact on these women's experiences as they move along the astrophysics trajectory. In some cases, like Ruby's, this gendering can act as an affordance. For instance, Ruby is an example of a student who rejects traditional forms of femininity and seeks out more stereotypical (and masculine) identity positions within the astrophysics community, as a strategy to be recognized as an insider to the culture. This finding, on the one hand, demonstrates a reification of the troubling positioning of stereotypical femininity outside of astrophysics culture, and, on the other hand, presents an interesting example of improvisation [28], where a positioning against femininity is used strategically to garner recognition in the field. This construction of gender does not challenge the dominant framing of physics fields as masculine or of femininity as "Other" [46] to physics fields, but it does trouble the 
binary-model assumption that masculinity or femininity maps neatly onto male or female, a model on which much research into women in physics is predicated [11].

Another reading of the problem of gender neutrality comes from Halberstam's [45] work on the construction of masculinity. Halberstam argues that masculinity is often not culturally viewed as performative, but rather is seen as a natural characteristic associated with the male sex. In this sense, the masculine culture of physics fields may be viewed as natural (since it is populated so highly by men) and thus masculinity is easily conflated with gender neutrality. The implication of this for women like Molly is that the gender neutral (or masculine) cultural model of the stereotypical astrophysicist can emerge as a constraint. Molly's narrative demonstrates that the implied gender neutrality of the stereotypical astrophysicist cultural model can render expressions of femininity (in)visible, and subject to microaggressions. Molly found ways to author herself as a recognizably competent astrophysicist in her research community, but still found herself frequently positioned as an outsider. In the stories of both Ruby and Molly, it is possible to see that doctoral students experience astrophysics in a context of social forces that can shape who gets recognized as successful or competent astrophysicists. Often, physics and related disciplines are characterized as separate or outside of social forces, but this has itself been interpreted as a "distinctive cultural feature" of physics [51] (p. 61), which Traweek describes as a "culture of no culture" [30]. In this sense, the predominance of the discourse of gender neutrality becomes distinctly problematic, as it obscures and normalizes the gendering of astrophysics [11].

\section{B. Pipeline problems}

The sociocultural framing employed in this study helps reveal important, though contradictory, lessons for researchers and policymakers interested in addressing the underrepresentation of women in astrophysics. These two case studies of women who move in and out of identification with the field of astrophysics challenge a persistent discourse about women who leave academic science fields through the so-called "leaky pipeline." This pipeline is generally conceived of as a narrow and linear trajectory, particularly associated with career paths that are identified as masculine [52]. In the pipeline, women and people of color are thought of as passive resources that fail to make it through the pipeline, rather than agents who make active choices about their career paths [52]. Critics of the pipeline model argue that it is wrongly depicted as unidirectional, orderly and rigid, and associated with traditional and academic career paths [53]. Recent work [54] presents a critique of the pipeline model that is significant for this study: pipeline research fails to look carefully at the contexts of disciplinary subculture in science (a critical point for work focused specifically on physics and astrophysics). Mendick and colleagues [54] suggest that the pipeline model narrowly defines what counts as persistence in science, and that this discourse of persistence into an academic career is fraught with elitism that goes unchallenged in current conceptions of the pipeline. The stories of Ruby and Molly give credence to this critique by demonstrating that doctoral work in astrophysics positions students on trajectories into very elite disciplines (with a very limited set of possible identities in academic fields). While research that explores factors related to the leaky pipeline at the upper levels of astrophysics (e.g., Ref. [4]) gives us a sense of the structural barriers to women in astrophysics, it does not account for the cultures of academic elitism fostered at this level, nor does it account for the intersection of gender, race, or class with these cultural factors.

The story of Ruby provides a concrete example that not all women experience gender or the gendering of physics cultures in the same way. Research that takes a binary deficit perspective [11] on women's experiences in astrophysics would miss the multiple facets of participation that students like Ruby use to navigate the figured world of astrophysics. Furthermore, policy initiatives designed to attract women to or retain them in astrophysics in ways that depend on normative constructions of gender would likely alienate students like Ruby. Additionally, while these case studies present narratives of identity trajectories that may otherwise be interpreted as leaks from the traditional science pipeline model, they also challenge the predictive power that gender has in that model [54]. The pipeline model that seeks to redress structural problems related to the gender gap does not address the problems that Ruby had in seeking to author an identity for herself in relation to the cultural model for competence. This cultural model might be characterized as elitist in the sense that it requires very specific forms of participation that entail not only acquiring the computing skills and physics knowledge required to persist in astrophysics, but also the social skills that only come with repeated participation in a collaborative process. Often, these kinds of skills form the unwritten rules of doctoral education that can be used to exclude certain individuals or groups of individuals, or prevent them from becoming full members, thereby maintaining the elitist nature of doctoral education [55]. These collaborative and communicative skills are critical to develop in a field that is characterized by increasingly large collaborative models for participation [56]. In another vein, despite Molly's experiences of microaggressions throughout her program, and the (in) visibility she experienced throughout her pregnancy, she otherwise had a strong relationship with her research team and advisor, and she persisted through to the postdoctoral level before turning on her pathway towards teaching.

In both cases, Ruby and Molly chose to enter physicsrelated teaching fields, where their identity trajectories as astrophysicists persisted, albeit in a different form. These 
cases provide two examples of identity trajectories that are not adequately represented by the traditional pipeline model for doctoral astrophysics, which predicts a common end point. Researchers have suggested a conceptual change in tracking the progress of girls and women through science-related fields, and have offered metaphors like pathways [57] and ecosystems [58] to frame the trajectories of young students who do not persist to research-related careers. The narratives presented here demonstrate a similar need to reframe the understanding of women's participation in doctoral astrophysics programs to account for agency and identity, and to broaden the current definitions of what counts as persistence in astrophysics.

\section{Identity trajectories}

The cases presented here demonstrate the need to document the unexpected resources and cultural models that students may draw upon as they navigate pathways through astrophysics that do not presuppose linearity. Thinking about movement along educational pathways as "identity trajectories" [9] allowed me to capture "forces" on individuals' trajectories that propel them "towards or away from science" (p. 831). Emerging from these two case studies are examples of different kinds of identity work that takes place in the figured world of astrophysics, and how this work constitutes a weaving in and out of "insider" identities [19]. For example, Ruby began her story by authoring herself as an insider to physics through a "good student identity" [59] (p. 404) early on, in response to the positive forms of recognition she received from peers. These sources of recognition seemed critical to Ruby's movement in and out of her identity constitution as an insider to astrophysics. At first, they served as a resource, "thickening" [60] her feelings of insiderness, but as she continued in her studies, these sources of recognition were no longer as accessible to Ruby and may have in fact hindered her ability to author herself as a good student. She often described not being able to avail herself of the resources available to good students, and therefore sought other unconventional means of recognition to maintain her insider trajectory. This raises concerns about how to support graduate students as they transition from a more classroom-based environment in undergraduate astrophysics programs to the more independent work of graduate studies. Ruby's case demonstrates that the resources that students are expected to make use of in doctoral astrophysics work are not always available to every student, and this may present a constraint for their identity work. The activities like Star Tea and Cosmic Coffee that are intended to promote social cohesion and collaborative learning, actually worked to accelerate Ruby's identity trajectory away from astrophysics because she could no longer draw on her identity as a good student in this context.

In contrast, Molly's case began with a positioning of herself as an outsider to astrophysics culture. Molly seemed to embrace this identity, and perhaps her desire not to "fit in" to astrophysics culture contributed to her resilience at times when she was not recognized as an insider. However, we see that Molly's trajectory was greatly facilitated by her use of the resources available in the figured world of astrophysics, which seemed to contribute to a thickening of her identity as an insider [60]. Instead, it was forces external to astrophysics culture that prompted a change in Molly's pathway through astrophysics. Ultimately, both doctoral students chose college-level teaching as the next direction in their pathways. For Ruby, teaching appeared to become a resource for recognition as an insider to astrophysics. For Molly, teaching emerged as a way for her to attain work-life balance, while still maintaining an insider status to astrophysics.

For both Ruby and Molly, questions arise about what it means for cultural change in astrophysics if doctoral students who do not fit the recognizable mold for an astrophysicist instead choose to enter teaching. Perhaps trajectories that lead to teaching provide an opportunity for cultural change early, at the college or high school level. There are scant studies in science or physics education research that explores how science identity trajectories are formed over long timescales; cf. Ref. [21]. What kind of a teacher does an astrophysicist become? How do identity trajectories as astrophysicists extend into the physics classroom? Further research into the identity trajectories of astrophysicists who enter teaching professions at all levels might explore how recognition operates in the figured worlds of their classrooms. Importantly, research might investigate how these teachers learn to recognize and support good students who, like themselves, may not fit neatly into the dominant cultural models produced in academic astrophysicists, or how physics teachers may unwittingly perpetuate the constructs of the world they "left behind."

\section{ACKNOWLEDGMENTS}

This research was funded by the Social Sciences and Humanities Research Council of Canada. I would like to thank the doctoral students and professors who participated in this study, particularly "Ruby" and "Molly." I would also like to thank Dr. Margaret Livingstone for her careful reading of this manuscript. Finally, I would like to acknowledge and thank the very helpful feedback given by the reviewers of this manuscript. 
[1] Statistiques sur les inscription des femmes en sciences et en génie à l'université entre 2007 et 2014. Chaire pour les femmes en sciences et en génie au Québec. (2017), accessed 11/05/17.

[2] S. Nicholson and P. Mulvey, Focus on astronomy enrollments and degrees, http://aip.org/statistics/reports/ astronomy-enrollments-and-degrees, accessed 11/05/17.

[3] R. Ivie, S. White, A. Ephraim, and G. Anderson, Women among physics and astronomy faculty, http://aip.org/sites/ default/files/statistics/faculty/womenfac-pa-10.pdf, accessed 11/05/17.

[4] R. Ivie, S. White, and R. Chu, Women's and men's career choices in astronomy and astrophysics., Phys. Rev. Phys. Educ. Res. 12, 020109 (2016).

[5] R. S. Barthelemy, M. McCormick, and C. Henderson, Gender discrimination in physics and astronomy: Graduate student experiences of sexism and gender microaggressions, Phys. Rev. Phys. Educ. Res. 12, 020119 (2016).

[6] A. Gonsalves, Gender and Discourses in doctoral physics, empirical dissertation, McGill University, 2010 (unpublished).

[7] A. J. Gonsalves, A. T. Danielsson, and H. Pettersson, Masculinities and experimental practices in physics: The view from three case studies, Phys. Rev. Phys. Educ. Res. 12, 020120 (2016).

[8] A. J. Gonsalves, 'Physics and the girly girl-there is a contradiction somewhere': doctoral students' positioning around discourses of gender and competence in physics, Cult. Stud. Sci. Educ. 9, 503 (2014).

[9] P. Jackson and G. Seiler, Science identity trajectories of latecomers to science. J. Res. Sci. Teach. 50, 826 (2013).

[10] R. Ivie and K. N. Ray, Women in physics and astronomy, 2005 http://www.aip.org/statistics/reports/women-physicsand-astronomy-2005.

[11] A. L. Traxler, X. C. Cid, J. Blue, and R. Barthelemy, Enriching gender in physics education research: A binary past and a complex future, Phys. Rev. Phys. Educ. Res. 12, 020114 (2016).

[12] A. T. Danielsson, Exploring woman university physics students 'doing gender' and 'doing physics', Gender Educ. 24, 25 (2012).

[13] J. Butler, Gender Trouble: Feminism and the Subversion of Identity (Routledge, New York, 1990).

[14] J. Butler, Performative acts and gender constitution: An essay in phenomenology and feminist theory, Theatre J. 40, 519 (1988).

[15] C. Paechter, Masculine femininities/feminine masculinities: Power, identities and gender, Gender Educ. 18, 253 (2006).

[16] B. A. Brown, J. M. Reveles, and G. J. Kelly, Scientific literacy and discursive identity: A theoretical framework for understanding science learning, Sci. Educ. 89, 779 (2005).

[17] Y. Engeström, Learning by Expanding: An ActivityTheoretical Approach to Developmental Research (Orienta-Konsultit, Helsinki, 1987).

[18] J. L. Lemke, Articulating communities: Sociocultural perspectives on science education, J. Res. Sci. Teach. 38, 296 (2001).
[19] J. Rahm, Youths' and scientists' authoring of and positioning within science and scientists' work, Cult. Stud. Sci. Educ. 1, 517 (2007).

[20] A. C. Barton, H. Kang, K. Tanner, T. B. O’Neill, J. Bautista-Guerra, and C. Brecklin, Crafting a future in science: Tracing middle school girls' identity work over time and space, Am. Educ. Res. J. 50, 37 (2013).

[21] H. B. Carlone, C. M. Scott, and C. Lowder, Becoming (less) scientific: A longitudinal study of students' identity work from elementary to middle school science, J. Res. Sci. Teach. 51, 836 (2014).

[22] K. P. Dabney and R. H. Tai, Female physicist doctoral experiences, Phys. Rev. ST Phys. Educ. Res. 9, 010115 (2013).

[23] H. Carlone and A. Johnson, Understanding the science experiences of successful women of colour: Science identity as an analytic lens, J. Res. Sci. Teach. 44, 1187 (2007).

[24] J. P. Gee, Identity as an analytic lens for research in education, Rev. Res. Educ. 25 (2000-2001).

[25] Z. Hazari, G. Sonnert, P. Sadler, and M.-C. Shanahan, Connecting high school physics experiences, outcome expectations, physics identity, and physics career choice: A gender study, J. Res. Sci. Teach. 47, 978 (2010).

[26] A. T. Danielsson. In the physics class: University physics students' enactment of class and gender in the context of laboratory work, Cult. Stud. Sci. Educ. 9, 477 (2014).

[27] A. Johnson, J. Brown, H. Carlone, and A. K. Cuevas, Authoring identity amidst the treacherous terrain of science: A multiracial feminist examination of the journeys of three women of color in science, J. Res. Sci. Teach. 48, 339 (2011).

[28] D. Holland, W. Lachicotte, D. Skinner, and C. Cain, Identity and Agency in Cultural Worlds (Harvard University Press, Cambridge, MA, 1998).

[29] J. P. Gee, An Introduction to Discourse Analysis: Theory and Method, 2nd ed. (Routledge, New York, 2010).

[30] S. Traweek, Beamtimes and Lifetimes: The World of High Energy Physicists (Harvard University Press, Cambridge, MA, 1988).

[31] M. Miles and A. M. Huberman, Qualitative Data Analysis (Sage Publications, Thousand Oaks, CA, 2014).

[32] K. Douglas, Impressions: African American first-year students' perceptions of a predominantly White university, J. Negro Educ. 67, 416 (1998).

[33] A. V. Sampson-Cordle, Exploring the relationship between a small rural school in Northeast Georgia and its community: An image-based study using participant-produced photographs (unpublished doctoral dissertation), University of Georgia, Athens (2001).

[34] C. Mitchell and J. Larkin, "Because you can be raped in this place...": Using photo-voice approaches to disrupt the silences around sexuality, the body, and HIV/AIDS [paper presented at the Dangers and Pleasures Revisited Conference), Cardiff, Wales (2004, June).

[35] D. Harper, An argument for visual sociology, in ImageBased Research: A Sourcebookfor Qualitative Researchers, edited by J. Prosser (Falmer Press, London, 1998), p. 24.

[36] D. Harper, Talking about pictures: A case for photo elicitation, Visual Studies 17, 13 (2002). 
[37] N. Gilbert, Researching Social Life (Sage Publications, Thousand Oaks, CA, 1993).

[38] S. Kvale, InterViews: An Introduction to Qualitative Research Interviewing (Sage Publications, Thousand Oaks, CA, 1996).

[39] E. G. Mishler, Storylines: Craftartists' narratives of identity (Harvard University Press, Cambridge, MA, 1999).

[40] J. P. Gee, Identity as an analytic lens for research in education, Rev. Res. Educ. 25 (2000-2001).

[41] W. Faulkner, 'Nuts and bolts and people': Gender-troubled engineering identities, Soc. Stud. Sci. 37, 331 (2007).

[42] J. Wajcman, Feminism Confronts Technology (The Pennsylvania State University Press, University Park, PA, 1991).

[43] L. Archer, E. Dawson, J. DeWitt, A. Seakins, and B. Wong, 'Science capital': A conceptual, methodological, and empirical argument for extending bourdieusian notions of capital beyond the arts, J. Res. Sci. Teach. 52, 922 (2015).

[44] B. Francis, L. Archer, J. Moote, J. de Witt, and L. Yeomans, Femininity, science, and the denigration of the girly girl, Brit. J. Soc. Educ. 38, 1097 (2017).

[45] J. Halberstam, Female masculinity (Duke University Press, Durham, 1998).

[46] M. Ong, Body projects of young women of color in physics: Intersections of gender, race, and science, Social problems 52, 593 (2005).

[47] M. Walker, Engineering identities, Br. J. Sociol. Educ. 22, 75 (2001).

[48] W. Faulkner, Doing gender in engineering workplace cultures: II. Gender in/authenticity and the in/visibility paradox, Eng. Stud. 1, 169 (2009).

[49] E. Kvande, 'In the belly of the beast': Constructing femininities in engineering organizations, Euro. J. of Women's Stud. 6, 305 (1999).
[50] F. Henwood, Engineering difference: Discourses on gender, sexuality and work in a college of technology, Gender Educ. 10, 35 (1998).

[51] S. Harding, Is Science Multicultural? Postcolonialisms, Feminisms, and Epistemologies (Indiana University Press, Bloomington, IN, 1998).

[52] H. Metcalf, Stuck in the pipeline: A critical review of STEM workforce literature, J. Educ. Info. Stud. 6, 2 (2010). Retrieved from https://escholarship.org/uc/item/ $6 z f 09176$

[53] Y. Xie and K. Shauman, Women in Science: Career Processes and Outcomes (Harvard University Press, Cambridge, 2003).

[54] H. Mendick, M. Berge, and A. Danielsson, A critique of the STEM pipeline: Young people's identities in Sweden and science education policy, Brit. J. Ed. Stud. 65, 481 (2017).

[55] J. Hadingham, The experience of becoming a $\mathrm{PhD}$, empirical dissertation, University of Witwatersrand, 2011 (unpublished).

[56] J. Adams, Collaborations: The rise of research networks, Nature (London) 490, 335 (2012).

[57] A. C. Barton, L. Dierking, D. Greenberg, L. Archer, E. Dawson, and A. Seakins, Pathways in Informal Science Learning: A Practice-Research Brief (King's College, London, UK, 2016).

[58] J. Lucena, Defending the Nation: U.S. Policymaking to Create Scientists and Engineers from Sputnik to the 'War against Terrorism' (University Press of America, Lanham, 2005).

[59] H. B. Carlone, The cultural production of science in reform-based physics: Girls' access, participation, and resistance, J. Res. Sci. Teach. 41, 392 (2004).

[60] D. Holland and J. Lave, History in Person (School of American Research Press, Santa Fe, NM, 2001). 